\title{
Linguistic correlates of pantomime recognition in aphasic patients ${ }^{1}$
}

\author{
N I L S R. VAR NEY \\ From the Benton Laboratory of Neuropsychology, Department of Neurology, University of Iowa \\ Hospital and Clinics, and the Psychology Service, Veterans Administration Hospital, Iowa City \\ Iowa, USA
}

SUM MARY Aphasic patients were given tests assessing pantomime recognition, reading comprehension, aural comprehension, and naming ability to determine whether defective pantomime recognition could be the result of a disturbance of symbolic thinking also affecting linguistic functioning. Defects in pantomime recognition always occurred in conjunction with reading defects of at least comparable severity, but reading defects sometimes occurred without comparable defects in pantomime recognition. The relationship of pantomime recognition with both aural comprehension and naming ability was significantly weaker than that between pantomime recognition and reading comprehension. The implications of the findings with regard to other nonverbal aphasic symptoms and the role of sensory modality factors are discussed.

Impaired pantomime recognition as a correlate of aphasia has been noted frequently since the midnineteenth century. Finkelnburg (1870) was the first to attribute this nonverbal defect to "asymbolia," a general disturbance of symbolic thinking in which verbal and nonverbal abilities are equally impaired. Similarly, Jackson (1878) and Head (1926) viewed pantomime recognition defects as being the result of a determinant which also affected linguistic functioning. Opposed to this point of view are authors (for example, Wernicke, 1874; Geschwind, 1965) who regard aphasia as a specific disturbance of conventional language operations whose determinants are functionally distinct from those affecting nonverbal abilities.

A study of pantomime recognition by Duffy et al. (1975) indicated that defects in this ability are frequent among aphasic patients, but very rare among nonaphasic patients with focal brain lesions. In addition, the performance of aphasic patients in pantomime recognition was found to be significantly correlated with naming ability, aural comprehension, and overall linguistic com-

\footnotetext{
1 This investigation was supported by Research Grant NS-00616 from the National Institute of Neurological and Communicative Disorders and Stroke.

Address for reprint requests: Dr Nils Varney, Psychology Service, Veterans Administration Hospital, Iowa City, Iowa 52240, USA.

Accepted 29 January 1978
}

petence. The authors concluded that pantomime recognition defects occurred at the "same general level of severity" as linguistic defects, and echoed Head in recommending that "an impairment inco" central symbolic activity" was at the root of all? aphasic symptoms.

Evidence supporting other viewpoints has also been obtained. Gainotti and Lemmo (1976) also found that impaired pantomime recognition was highly variable among aphasic subjects, with individuals performing at all levels of competence. Zangwill (1964) and Alajouanine and Lhermitte (1964) have described severely aphasic patients in whom nonverbal communicative abilities appeared to be intact. The latter authors concluded that "aphasia appears not to bear any necessary relationship to the presence or absence of extralinguistic disorders."

Although these findings suggest that defects in pantomime recognition are essentially aphasic in nature, they do not provide any clear evidence as to whether the determinant of this nonverbal defect has a comparable effect on linguistic functioning. This would require finding that impaired pantomime recognition is always associated with a comparably severe impairment in linguistic functioning. The purpose of the present study was to investigate the relationship between pantomime recognition and linguistic functioning to determine 
whether or not such a relationship exists. Of particular interest in this regard were two receptive language abilities, aural comprehension and reading comprehension. The former was of interest because most previous studies of nonverbal aphasic symptoms (for example, constructional apraxia, impaired sorting, defective sound recognition) have indicated that these defects are closely associated with defects in aural comprehension (De Renzi et al., 1965; Vignolo, 1969; Benton, 1973). However, because pantomime recognition is a visually mediated ability, it was suspected that reading comprehension might be more directly relevant.

\section{Subjects and methods}

Two groups of patients, aphasic and control, were selected from patient populations at University Hospitals and the Veterans Administration Hospital, Iowa City, Iowa. Inclusion in either group was restricted to patients who were right handed, who had at least eight years of education, who had no history of psychiatric hospitalisation or mental deficiency dating back to childhood, and who were physically able to undergo the $60 \mathrm{~min}$ utes of testing required in the study.

The aphasic group comprised 40 patients who had cerebral lesions confined to the left hemisphere and who performed below the fifth percentile on the standardised test of expressive language functioning described below. The presence of lateralised cerebral lesions was established by neurological examination and the results of radiographic tests-that is, brain scan, angiogram, computer axial tomography-when the latter were available. Twenty-six patients had vascular lesions, 12 had neoplastic lesions, and two had lesions from head trauma.

The control group comprised 20 patients who were hospitalised for non-neurological reasons, and who had no evidence of past or present neurological disease. This group was used in the standardisation of the Pantomime Recognition Test.

\section{TESTS}

\section{Linguistic function}

Three tests of the Multilingual Aphasia Examination (Benton, 1969) were used to assess specific linguistic functions in the aphasic patients. For each test, a performance was regarded as defective if it fell below the fifth percentile of norms previously established for each test.

Visual naming This test assessed oral expressive vocabulary and naming ability. The 30 test items were drawings of common objects which the subject was required to name appropriately. One or two points were given for each response given within specified time limits. Based on the test's norms, scores at or below 42 correct were regarded as defective.

Aural comprehension This test required subjects to point to drawings of objects named or described by the examiner. Four choices were available on each of 21 trials, and two points were given for each correct pointing response. Based on the test's norms, scores at or below 33 correct were regarded as defective.

Reading comprehension This 20 item test required subjects to point to drawings of objects whose name or description he had read. Stimulus words were printed in half-inch type on $5 \times 8$ inch white cards. Four choices were available on each trial, and one point was given for each correct pointing response. Based on the test's norms, scores at or below 17 correct were regarded as defective.

\section{Pantomime recognition}

This test involved pantomimes that depicted the use of common objects (for example, comb, spoon, pen) and which were recorded on videotape for presentation on a television monitor. The patient's ability to recognise these pantomimes was assessed by requiring him to point to a drawing of the object whose use was pantomimed. On each of the four practice trials and 30 test trials, subjects chose from among four drawings. Response foils were modelled after those employed by Vignolo (1969) in his study of sound recognition such that a subject's choices always included the following: (1) correct choice-the object whose use was pantomimed (for example, a saw); (2) semantic foil-an object belonging to the same class of objects as the stimulus (for example, an axe); (3) regular foil-an object whose use was pantomimed elsewhere in the test (for example, a pen); (4) odd foil-an object whose use is unsuited for pantomime (for example, an oil well).

The test proved to be relatively easy for control patients, with seven $(34 \%)$ obtaining perfect scores of 30 correct. The poorest performance, made by two controls, was 26 . Performance below this level (that is, 25 and below) were regarded as defective.

\section{Results}

The performance of the aphasic patients on the pantomime recognition test ranged from errorless scores of 30 to a grossly defective score of 10 , and 14 aphasics $(35 \%)$ made defective scores of 25 and below (that is, below the worst control subject). A 
similarly wide distribution of scores was observed for reading comprehension (from 20 to 5 correct) and aural comprehension (from 42 to 9 correct). Eighteen aphasics $(45 \%)$ failed reading comprehension, and $26(65 \%)$ failed aural comprehension. The visual naming test, which was a criterion for inclusion in the aphasic group, was failed by all $\mathbf{4 0}$ patients, and for $13(32 \%)$ this was the only test which was failed. Based on the performances of the aphasic patients, the split-half reliability coefficient of the pantomime recognition test was 0.84 , that of reading comprehension was 0.91 , that of aural comprehension was 0.89 , and that of visual naming was 0.90 .

Intercorrelations among the four test performances for the aphasic patients are shown in Table 1. All of the six correlation coefficients obtained proved to be positive and highly significant. Comparison of individual between-test correlations showed that the correlation between pantomime recognition and reading comprehension was significantly closer than that between pantomime recognition and either aural comprehension $(\mathrm{t}=4.1)$ or visual naming $(t=3.9)$. Thus, reading comprehension proved to be the linguistic ability most closely associated with pantomime recognition.

It was also found that the correlation between pantomime recognition and reading comprehension was significantly closer than that between reading comprehension and either of the linguistic tests, aural comprehension $(t=2.5)$ and visual naming $(t=3.5)$. No other pair of related correlation coefficients differed significantly.

Table 1 Test intercorrelation matrix

\begin{tabular}{llll}
\hline & $\begin{array}{l}\text { Pantomime } \\
\text { recognition }\end{array}$ & $\begin{array}{l}\text { Reading } \\
\text { comprehension }\end{array}$ & $\begin{array}{l}\text { Aural } \\
\text { comprehension }\end{array}$ \\
\hline Reading comprehension & .87 & & \\
Aural comprehension & .61 & .71 & .65 \\
Visual naming & .60 & .62 & .62 \\
\hline
\end{tabular}

A close relationship between pantomime recognition and reading comprehension was also indicated by the contingencies of intact and defective test performances shown in Table 2. As can be seen from the table, all 14 of the aphasic patients who performed at a defective level on the pantomime recognition test also failed reading comprehension, and all 22 aphasics who showed intact reading comprehension performed adequately on pantomime recognition. There were, however, four aphasic patients who performed at a defective level on reading comprehension while passing pantomime recognition. Only 13 of the 26 aphasic patients who showed significant defects on aural comprehension failed pantomime recognition, and one of the aphasics who failed pantomime recognition showed intact aural comprehension.

Table 2 Distribution of intact and defective test performances-results from 40 aphasic patients

\begin{tabular}{lcc}
\hline & Pantomime recognition performance \\
\hline Linguistic test performance & PR intact & PR defective \\
\hline RC intact, AC intact $(n=13)$ & 13 & 0 \\
RC defective, AC intact $(n=1)$ & 0 & 1 \\
RC intact, AC defective $(n=9)$ & 9 & 0 \\
RC defective, AC defective $(n=17)$ & 4 & 13
\end{tabular}

$\mathbf{P R}=$ pantomime recognition, $\mathbf{R C}=$ reading comprehension, $\mathbf{A C}=$ aural comprehension.

The relationship between pantomime recognition and linguistic test performance was explored further to determine whether defects in these abilities occurred at comparable levels of severity. To accomplish this, the raw score performances of each patient on each test were converted to standard scores, based on the number of standard deviations an individual performance was above or below the criterion for defective performance. By this method, an individual patient's performances on different tests could be directly compared. The distribution of the obtained difference scores is shown in Table 3.

Table 3 Distribution of difference scores for comparisons of pantomime recognition and linguistic tests-results from 40 aphasic patients involving 120 individual test comparisons

\begin{tabular}{lccc}
\hline Extent of difference & $R C$ & $A C$ & $V N$ \\
\hline PR superior by more than 2 SDs & 0 & 1 & 11 \\
PR superior by 1 or 2 SDs & 5 & 15 & 14 \\
PR differs by less than 1 SD & 35 & 23 & 15 \\
PR inferior by 1 to 2 SDs & 0 & 1 & 0 \\
PR inferior by more than 2 SDs & 0 & 0 & 0 \\
\hline PR = pantomime recognition, RC=reading comprehension, $\mathbf{A C}=$ \\
aural comprehension, VN=visual naming.
\end{tabular}

It was found that 47 of the individual test comparisons $(39 \%)$ involved differences of one or more standard deviations. In 46 of these, pantomime recognition was the superior test performance. The remaining case was a patient who performed 1.8 SD better on aural comprehension than in pantomime recognition. Reading comprehension was never more than $0.6 \mathrm{SD}$ better than pantomime recognition. In addition, 35 aphasic patients' performances on pantomime recognition and reading comprehension differed by less than $1 \mathrm{SD}$, and 32 differed by 0.5 SD or less. By comparison, there were 17 patients for whom panto- 
mime recognition and aural comprehension differed by 1 SD or more, and 27 for whom pantomime recognition and visual naming also differed by $1 \mathrm{SD}$ or more.

\section{Discussion}

The results of the study clearly indicate a close relationship between pantomime recognition and reading comprehension. The correlation between these visually mediated test performances was impressively high, and it was significantly closer than other between-test correlations involving either test. In addition, impaired pantomime recognition was always associated with reading comprehension defects of at least comparable severity. This finding demonstrates that the determinant of defective pantomime recognition had a comparable effect on reading comprehension.

At the same time, the results also indicated that defects in reading comprehension were not always associated with defects in pantomime recognition, and that in some instances, reading was significantly more impaired than pantomime recognition. Furthermore, two Wernicke aphasics, both with severe defects in all aspects of linguistic functioning, showed clearly intact pantomime recognition. It would appear, therefore, that intact pantomime recognition is a necessary, but not sufficient, condition for intact reading. This implies that there are at least two determinants of reading defects: a supralinguistic impairment (asymbolia?) which also affects nonverbal abilities, and a specific linguistic impairment which only affects reading comprehension.

It is also apparent from the results that the relationship between pantomime recognition and aural comprehension was weaker and less predictable than that between pantomime recognition and reading comprehension. Not only was the correlation between these tests smaller than that between pantomime recognition and reading comprehension, but half of the aphasics with impaired aural comprehension showed intact pantomime recognition, and one aphasic with impaired pantomime recognition performed adequately in aural comprehension. These findings suggest that there may be no essential relationship between defects in pantomime recognition and aural comprehension.

These conclusions differ substantially from those of the study of pantomime recognition by Duffy et al. (1975). Based on their finding of significant correlation between pantomime recognition and various aspects of linguistic functioning in aphasic patients, the authors concluded that aphasics "typically demonstrated" pantomime recognition defects which were at the "same general level of severity" as linguistic defects. Although the correlation coefficients obtained in the present study are similar in magnitude to those reported by Duffy et al., pantomime recognition defects were observed in only a minority of the present sample of aphasics, and some severely aphasic patients showed intact pantomime recognition. The findings also fail to support Alajouanine and Lhermitte (1964) who, based on similar observations, concluded that verbal and nonverbal aphasic symptoms involved functionally distinct determinants. For this view to have been supported, it would have been necessary to find aphasic subjects who performed significantly worse on pantomime recognition than on reading comprehension.

In many respects, the present findings are most similar to those reported by Vignolo (1969) in his investigation of sound recognition in aphasia. Like impaired pantomime recognition, impaired sound recognition is observed almost exclusively in aphasic patients. Vignolo found that this aurally mediated nonverbal symbolic defect was closely associated with impaired aural verbal comprehension, and he concluded that a single determinant was responsible for both types of defects. Thus, defects in sound recognition and pantomime recognition appear to be closely associated with defects in linguistic abilities mediated in the same sensory modality. If this conclusion is correct, then it may also be suggested that there are independent sensory modality factors affecting each of these nonverbal abilities that are analogous to the "modality specific" factors that have been found with regard to naming (Spreen et al., 1966) and language comprehension-that is, pure alexia, pure word deafness.

I would like to express my gratitude to Dr A. L. Benton for his advice and guidance during the course of this investigation.

\section{References}

Alajouanine, T., and Lhermitte, F. (1964). Non-verbal communication in aphasia. In Disorders of Language. Edited by A. De Reuck and M. O'Conner. Little Brown \& Company: Boston.

Benton, A. L. (1969). Development of a multilingual aphasia battery: progress and problems. Journal of the Neurological Sciences, 9, 39-48.

Benton, A. L. (1973). Visuoconstructive disability in patients with cerebral disease: its relationship to side of lesion and aphasic disorder. Documenta Ophthalmologica, 34, 67-76.

De Renzi, E., Faglioni, P., Savoyardo, M., and Vignolo, L. (1965). The influence of aphasia and 
hemispheric side of cerebral lesions on abstract thinking. Cortex, 2, 399-420.

Duffy, R., Duffy, J., and Pearson, K. (1975). Pantomime recognition in aphasic patients. Journal of Speech and Hearing Research, 18, 115-132.

Finkelnburg, F. (1870). Niederrheinische Gesellschaft, Sitzung vom. 21 Mai. Cited in Aphasia and Kindred Disorders of Speech. Edited by H. Head. Cambridge University Press: London.

Gainotti, G., and Lemmo, M. (1976). Comprehension of symbolic gestures in aphasia. Brain and Language, 3, 451-460.

Geschwind, N. (1965). Disconnection syndromes in animals and man. Brain, 88, 237-294.

Head, H. (1926). A phasia and Kindred Disorders of
Speech. Cambridge University Press: London.

Jackson, H. (1878). On afflictions of speech from disease of the brain. Brain, 1, 304-330.

Spreen, O., Benton, A. L., and Van Allen, M. (1966). Dissociation of visual and tactile naming in amnesic aphasia. Neurology (Minneapolis), 8, 807-814.

Vignolo, L. (1969). Auditory agnosia: a review and report of recent evidence. In Contributions to Modern Clinical Neuropsychology. Edited by A. L. Benton. Aldine: Chicago.

Wernicke, C. (1874). Der Aphasiche Symptomencomplex. Cohen and Weigert: Breslau.

Zangwill, O. (1964). Intelligence in aphasia. In Disorders of Language. Edited by A. De Reuck and M. O'Conner. Little Brown and Company: Boston. 